\title{
CaIPF7817 is involved in the regulation of redox homeostasis in Candida albicans
}

\author{
Jian-Hui Jia ${ }^{\text {b,1 }}$, Yan Wang ${ }^{\text {a,1 }}$, Yong-Bing Cao ${ }^{\text {a }}$, Ping-Hui Gao ${ }^{\text {a }}$, Xin-Ming Jia ${ }^{\text {a }}$, \\ Zhi-Ping Ma ${ }^{\mathrm{b}}$, Yong-Gang Xu ${ }^{\mathrm{a}}$, Bao-Di Dai ${ }^{\mathrm{b}}$, Yuan-Ying Jiang ${ }^{\mathrm{a}, \mathrm{b}, *}$ \\ a Department of Pharmacology, School of Pharmacy, Second Military Medical University, 325 Guohe Road, Shanghai 200433, PR China \\ ${ }^{\mathrm{b}}$ Department of Pharmacology, School of Life Science and Biopharmacology, Shenyang Pharmaceutical University, Shenyang 110016, PR China
}

Received 14 May 2007

Available online 22 May 2007

\begin{abstract}
CaIPF7817, a functionally unknown gene in Candida albicans, was suggested to be involved in the redox system previously, but its exact role is unknown. In this study, ipf7817 null mutant was generated with the URA-blaster method. After the deletion of CaIPF7817, intracellular levels of reactive oxygen species were significantly increased; mitochondrial membrane potential, a direct indicator of mitochondrial function, was elevated; some important redox-related genes, including GLR1, SOD2, and TRR1, were up-regulated; and the GSH/GSSG ratio was raised. These changes indicated that CaIPF7817 played important roles in the regulation of redox homeostasis in C. albicans.
\end{abstract}

(c) 2007 Elsevier Inc. All rights reserved.

Keywords: Candida albicans; CaIPF7817; Redox; Gene disruption

Candida albicans, the major human fungal pathogen, causes a range of disorders from mild infections to lifethreatening diseases $[1,2]$. Nowadays, with the widespread use of immunosuppressive therapy, infections by C. albicans have become more common and more studies about this pathogen are needed [3].

Like other living cells, C. albicans cells generate various oxidative agents, such as reactive oxygen species (ROS), from the mitochondrial respiratory chain in the normal aerobic metabolism process. ROS, such as superoxide anions, hydrogen peroxide, and hydroxyl radicals, can damage many of the cellular components [4-6]. Therefore, regulation of the redox homeostasis is important for cellular functions $[7,8]$. To maintain intracellular redox homeostasis in C. albicans, a series of regulation mechanisms are

\footnotetext{
* Corresponding author. Address: Department of Pharmacology, School of Pharmacy, Second Military Medical University, 325 Guohe Road, Shanghai 200433, PR China.

E-mail address: jiangyuanying@126.com (Y.-Y. Jiang).

${ }^{1}$ These authors contributed equally to this work.
}

involved [9], which require the participation of numerous genes [10]. However, our knowledge about the redoxrelated genes in $C$. albicans is limited today.

CaIPF7817, a potential redox-related gene probably encoding a $\mathrm{NAD}(\mathrm{P}) \mathrm{H}$ oxidoreductase, was investigated in this work. This gene was found highly up-regulated during oxidative stress [11,12]. Here, we studied the roles of CaIPF7817 in cellular redox regulation by constructing CaIPF7817 deletion mutants through deletion of both copies of the gene using the URA-blaster method and measuring various redox-related properties of these mutants.

\section{Materials and methods}

Strains, culture, and growth of C. albicans. Candida albicans strains used in this study were listed in Table 1 . The strains were cultured at $30^{\circ} \mathrm{C}$ in YPD medium ( $1 \%$ Difco yeast extract, $2 \%$ Difco peptone, $2 \%$ dextrose) or YPD medium supplemented with $0.005 \%$ uridine (Sigma, USA). Agar $(1.5 \%)$ was added to prepare the solid media. The URA3 transformants were selected on MIN ( $2 \%$ glucose, $0.67 \%$ Difco yeast nitrogen base without amino acids) agar plates supplemented with $25 \mu \mathrm{g} / \mathrm{mL}$ histidine. The ura 3 auxotrophs were obtained on MIN agar plates containing $0.1 \%$ 
Table 1

Candida albicans strains and plasmids used in this study

\begin{tabular}{|c|c|c|c|}
\hline & Parent & Genotypes & Reference \\
\hline \multicolumn{4}{|l|}{ Strains } \\
\hline RM1000 & RM100 & $\begin{array}{l}\text { ura34::imm434/ura34::imm434 } \\
\text { his1::hisG/his1::hisG }\end{array}$ & Negredo et al. (1997) \\
\hline $\mathrm{JH} 1 \mathrm{U}$ & RM1000 & $\begin{array}{l}\text { ipf78174::hisG-URA3-hisG/IPF7817 } \\
\text { ura3 }:: \text { imm434/ura3 }:: \text { imm434 } \\
\text { his1::hisG/his1::hisG }\end{array}$ & This work \\
\hline JH1 & JH1U & $\begin{array}{l}\text { ipf78174::hisG/IPF7817 } \\
\text { ura3 }: \because \text { imm434/ura3 } \because: \text { imm } 434 \\
\text { hisl }: \because \text { hisG/his1 }: \text { hisG }\end{array}$ & This work \\
\hline JH2U & JH1 & $\begin{array}{l}\text { ipf78174::hisG/ipf78174::hisG-URA3-hisG } \\
\text { ura3 } 1:: \text { imm434/ura3 } \because: \text { imm } 434 \\
\text { his1 }: \text { hisG/his1 }: \because \text { hisG }\end{array}$ & This work \\
\hline $\mathrm{JH} 2$ & $\mathrm{JH} 2 \mathrm{U}$ & $\begin{array}{l}\text { ipf78174::hisG/ipf78174::hisG } \\
\text { ura3 }: \because \text { imm434/ura3 }: \because \text { imm } 434 \\
\text { his1 }:: \text { hisG/his1 }: \text { hisG }\end{array}$ & This work \\
\hline JHEXP & $\mathrm{JH} 2$ & ipf78174::hisG/ipf78174::hisG::IPF7817 & This work \\
\hline \multicolumn{4}{|l|}{ Plasmids } \\
\hline p5921 & & Contains hisG-URA3-hisG cassette & Fonzi et al. (1993) \\
\hline p196 & & Contains the full-length of $I P F 7817$ gene & This work \\
\hline p196U & & hisG-URA3-hisG inserted into IPF7817 gene & This work \\
\hline pCaEXP & & Expression vector & Umeyama et al. [15] \\
\hline pCaEXP-7817 & & Expression vector containing $I P F 7817$ gene & This work \\
\hline
\end{tabular}

5-fluoroorotic acid (5-FOA; Lancaster, USA) and $50 \mu \mathrm{g} / \mathrm{mL}$ uridine. Escherichia coli DH5 $\alpha$ strain was grown in LB medium $(0.5 \%$ Difco yeast extract, $1 \%$ Difco Bacto tryptone, $0.5 \% \mathrm{NaCl}$ ) at $37^{\circ} \mathrm{C}$.

The C. albicans strains were grown in the YPD medium at $30^{\circ} \mathrm{C}$ under constant shaking $(200 \mathrm{rpm}) . \mathrm{OD}_{600}$ measurement at specific time points was used to monitor the growth of $C$. albicans cells.

Disruption and reintroduction of CaIPF7817. The plasmids used in this study were listed in Table 1 . The plasmid p196 was derived from the C. albicans cDNA library by integrating into the vector pBSK [13]. It contained the whole ORF of CaIPF7817 gene. To construct the ipf7817 null mutant of C. albicans, CaIPF7817 was disrupted by the method described by Fonzi and Irwin [14]. The URA-blaster cassette, derived from plasmid p5921 by BgIII and BamHI digestion, was inserted into the BglII site of p196 to yield plasmid $\mathrm{p} 196 \mathrm{U}$. The plasmid $\mathrm{p} 196 \mathrm{U}$ was propagated in E. coli $\mathrm{DH} 5 \alpha$ and linearized by PvuII to yield a $5.34 \mathrm{~kb}$ Aipf7817::hisG::URA3::hisG fragment, which was used to transform C. albicans strain RM1000. To reintroduce CaIPF7817 into the null strain, the whole CaIPF7817 gene was PCR amplified with Pyrobest DNA polymerase (TaKaRa) using primer 5'-GGTCGGAT CCATGACAGTTCCATACCAAGT-3' and $5^{\prime}$-TCGG CTGCAGCTAATTTACTTCTAAT TTAG- $3^{\prime}$. After being purified, the PCR product was cloned into the integrative expression vector $\mathrm{pCaEXP}[15]$ to generate the recombinant plasmid pCaEXP-7817. After sequencing, pCaEXP-7817 was linearized and used to transform the null JH2 strain to yield the JHEXP strain. Lithium acetate method was used for the transformation $[16,17]$.

Isolation of C. albicans DNA and Southern hybridization analysis. The small-scale isolation of DNA from C. albicans was performed as described previously [16]. Approximately $5 \mu \mathrm{g}$ genomic DNA was digested with PstI and SphI and then separated on a 1\% agarose gel. The separated DNA fragments were transferred onto nylon membranes, and Southern hybridization was performed using DIG labeling and detection kit (Roche). The 408 bp hybridization probe was PCR amplified with Pyrobest polymerase (TaKaRa) using the primers $5^{\prime}$-TCTGGAAGGATGA ACAAGCA-3' and 5'-CAAAACTGCCACCGTATTCA- $3^{\prime}$ from the genomic DNA of RM1000.

Measurement of ROS levels. Intracellular levels of ROS were measured with DCFH-DA $\left(2^{\prime}, 7^{\prime}\right.$-dichlorodihydro-fluorescein diacetate, Molecular Probes, USA) [18]. Briefly, C. albicans cells cultured in YPD medium at the exponential growth phase $\left(\mathrm{OD}_{600}=0.5\right)$ were collected by centrifugation $\left(3000 \mathrm{~g}, 5 \mathrm{~min}, 4^{\circ} \mathrm{C}\right)$ and washed three times with PBS. The cells were subsequently resuspended in PBS $\left(\mathrm{OD}_{600}=1.0\right)$. DCFH-DA was added to the final concentration of $20 \mu \mathrm{g} / \mathrm{mL}$. Cell suspensions were incubated at $30^{\circ} \mathrm{C}$ with shaking $(200 \mathrm{rpm})$ for $30 \mathrm{~min}$. At specified intervals, $100 \mu \mathrm{l}$ cell suspensions was transferred to the wells of a flat-bottom microplate (BMG Microplates, 96 well, Black) to measure fluorescence intensity on a FLUOstar fluorometer (BMG labtechnologies, Germany) with excitation at $485 \mathrm{~nm}$ and emission at $520 \mathrm{~nm}$. Triplicate experiments were conducted to generate a mean value.

Measurement of mitochondrial membrane potential $\left(\Delta \psi_{m}\right)$. Candida albicans cells from YPD cultures $\left(\mathrm{OD}_{600}=0.5\right)$ were collected by centrifugation $\left(3000 \mathrm{~g}, 5 \mathrm{~min}, 20^{\circ} \mathrm{C}\right)$ and washed three times with PBS. The cells were subsequently resuspended in PBS $\left(3 \times 10^{6}\right.$ cells $\left./ \mathrm{mL}\right)$. After treatment with

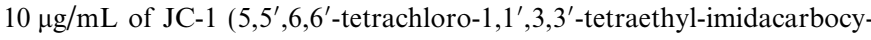
anine iodide, Molecular Probes, USA) in the dark at $30^{\circ} \mathrm{C}$ for $20 \mathrm{~min}$, cells were washed twice with PBS and analyzed on a FLUOstar fluorometer with excitation at $490 \mathrm{~nm}$ and emission at 530 and $590 \mathrm{~nm}$, respectively [19]. $\Delta \psi_{\mathrm{m}}$ was determined by the ratio of fluorescence intensity at $590 \mathrm{~nm}$ to that at $530 \mathrm{~nm}$.

$R N A$ isolation and real-time RT-PCR. RNA isolation and real-time RT-PCR were performed as described previously [11]. Triplicate experiments were performed with the Chromo4 Real-Time PCR System (BioRad, USA). SYBR Green I (TaKaRa) was used to visualize and monitor the amplified products in real time. Gene-specific primers were designed according to the manufacturer's protocol. Primers for SOD2 were $5^{\prime}$-CAG CACTATCGGAAGTAAC TC- $3^{\prime}$ and $5^{\prime}$-GGCATGTTATCATACTGG AAGG-3'; primers for GLRI were 5'-GCTCATCTAAGTCATTGTGA CC- $3^{\prime}$ and $5^{\prime}$-GCTGGACCAGAAGAAAAAGTTG- $3^{\prime}$; primers for $T R R 1$ were $5^{\prime}$-ACATTCCAAGGCAGCCATAC- ${ }^{\prime}$ and $5^{\prime}$-AGACCGAC GAAGCTGGTTAC- $3^{\prime}$; primers for $18 S$ rRNA were $5^{\prime}$-TCTTTCTTGA TTTTGTGGGTGG- $3^{\prime}$ and $5^{\prime}$-TCGATAGTCCCTCTAAGAAGTG-3'. The change in fluorescence of SYBR Green I dye in every cycle was monitored by the Opticon software (Bio-Rad, USA), and the threshold cycle $\left(C_{\mathrm{T}}\right)$ above background for each reaction was calculated. The $C_{\mathrm{T}}$ value of $18 \mathrm{~S}$ rRNA was subtracted from that of the gene of interest to obtain a $\Delta C_{\mathrm{T}}$ value. The $\Delta C_{\mathrm{T}}$ value of an arbitrary calibrator (i.e. sample of parental strain RM1000) was subtracted from the $\Delta C_{\mathrm{T}}$ value of each sample to obtain a $\Delta \Delta C_{\mathrm{T}}$ value. The gene expression level relative to the calibrator was expressed as $2^{-\Delta \Delta C_{\mathrm{T}}}$.

Colorimetric determination of reduced and oxidized glutathione. Candida albicans cells from YPD cultures $\left(\mathrm{OD}_{600}=0.5\right)$ were collected by centrifugation $\left(9000 \mathrm{~g}, 1 \mathrm{~min}, 4^{\circ} \mathrm{C}\right)$ and washed once with PBS. The cells were subsequently resuspended in the buffer for lyticase. Lyticase (Sigma, USA) 
was added according to the manufacturer's protocol. After incubating at $30{ }^{\circ} \mathrm{C}$ for $30 \mathrm{~min}$, the lysate was clarified by centrifugation $(9000 \mathrm{~g}, 5 \mathrm{~min}$, $4{ }^{\circ} \mathrm{C}$ ) and the supernatant was used to determine total free glutathione. Total glutathione was determined with the GSH and GSSG Assay Kit (Beyotime, PR China) [20,21]. Colorimetric determination was conducted using a MultiskanMK30 microplate reader (Labsystems, Finland).

Statistical analysis. Student's $t$ test was used to determine the statistical significance between experimental groups. Difference was considered significant if the $P$ value was less than 0.05 .

\section{Results}

\section{Generation and characterization of ipf7817 mutants}

To investigate the roles of CaIPF7817 in C. albicans, we disrupted the two alleles of CaIPF7817 sequentially in RM1000 strain using the URA-blaster method and 5-FOA selection, yielding the following strains: the $\mathrm{Ura}^{+}$ipf7817/ IPF7817 strain (JH1U), $\mathrm{Ura}^{-}$ipf7817/IPF7817 strain (JH1), $\mathrm{Ura}^{+}$ipf7817lipf7817 strain (JH2U), and $\mathrm{Ura}^{-}$ ipf7817/ipf7817 strain (JH2) (Table 1). The strategy to disrupt both copies of CaIPF7817 was depicted in Fig. 1A. Southern blot analysis of DNA from the constructed strains showed that the recombination patterns agreed with our expectation (Fig. 1B).

To further characterize the generated mutants, the growth curves of RM1000, JH1, and JH2 were obtained. They showed identical growth rates. To investigate if other deletions were introduced into the $C$. albicans DNA, CaIPF7817 was reintroduced into $\mathrm{JH} 2$ using the plasmid pCaEXP-7817 to generate the JHEXP strain. PCR analysis confirmed that CaIPF7817 was integrated into the RP10 locus in JHEXP strain. The growth rates of the RM1000 strain and JHEXP strain were similar, suggesting there was no other gene disruption that affected the growth of C. albicans.

\section{Increased intracellular ROS levels in ipf7817 mutants}

In order to study the roles of CaIPF7817 in intracellular redox balance, changes in intracellular levels of ROS after CaIPF7817 disruption were examined with the fluorescent dye DCFH-DA [22]. The changes of levels of ROS were monitored for $48 \mathrm{~h}$. Striking increases of intracellular ROS level were observed in ipf7817 null mutants. After $36 \mathrm{~h}$, the levels of ROS in $\mathrm{JH} 2 \mathrm{U}$ and $\mathrm{JH} 2$ were nearly five times that in the parental strain RM1000. In the heterozygote strain $\mathrm{JH} 1$ and $\mathrm{JH} 1 \mathrm{U}$, intracellular ROS levels were also elevated. In the JHEXP strain that CaIPF7817 was reintroduced, the change of ROS levels was similar with that in the parental strain RM1000 (Fig. 2).

\section{Increased mitochondrial membrane potential in ipf 7817 mutants}

Mitochondria are the main generator of intracellular ROS. We therefore investigated the effects of CaIPF7817 on mitochondria function by measuring $\Delta \psi_{\mathrm{m}}$, the direct

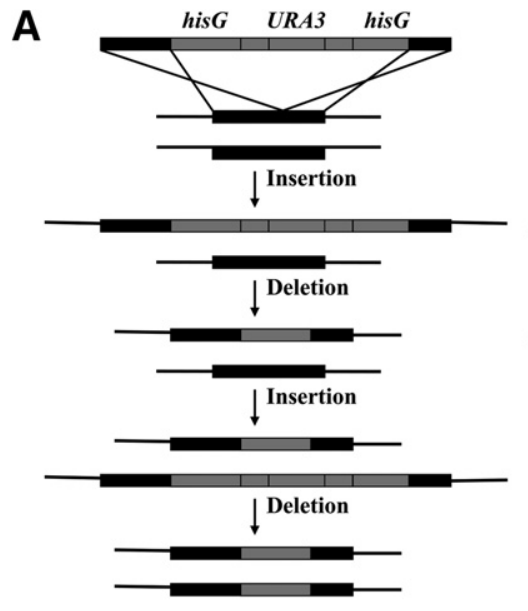

RM1000 Parent strain

$u^{\prime a} / u^{*}$

JH1U Heterozygous mutant $\mathrm{URA}^{+} / \mathrm{ura}^{-}$

JH1 Heterozygous mutant $\mathrm{ura}^{-1} \mathrm{ura}^{-}$

JH2U Homozygous mutant ${\text { ura } / U_{R A}^{+}}^{+}$

JH2 Homozygous mutant $u r a^{-} / u r a^{-}$

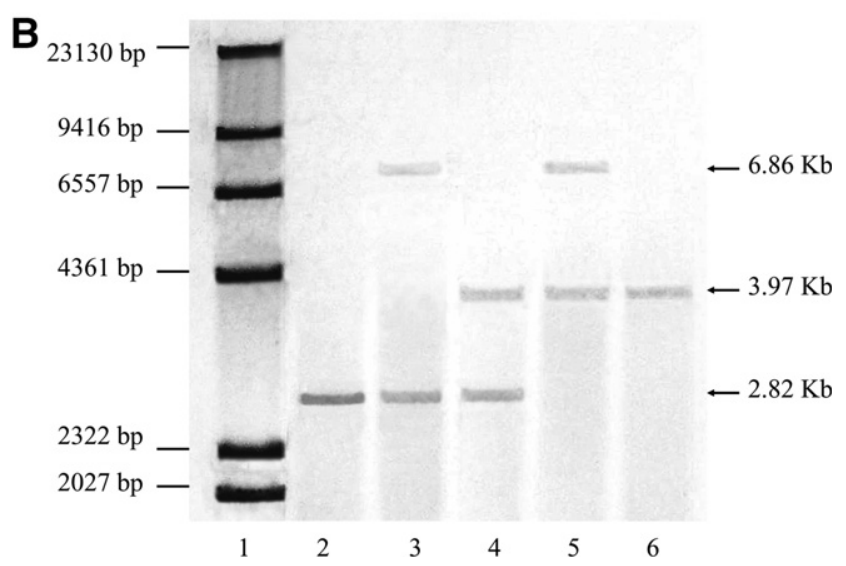

Fig. 1. Deletion of CaIPF7817 in C. albicans. (A) A diagram of the strategy to disrupt both copies of CaIPF7817. (B) Southern blot analysis on the genomic DNA digested with PstI and SphI. Lane 1, DNA molecular weight marker II, DIG-labeled (Roche); lane 2, RM1000; lane 3, JH1U; lane 4, JH1; lane 5, JH2U; lane 6, JH2.

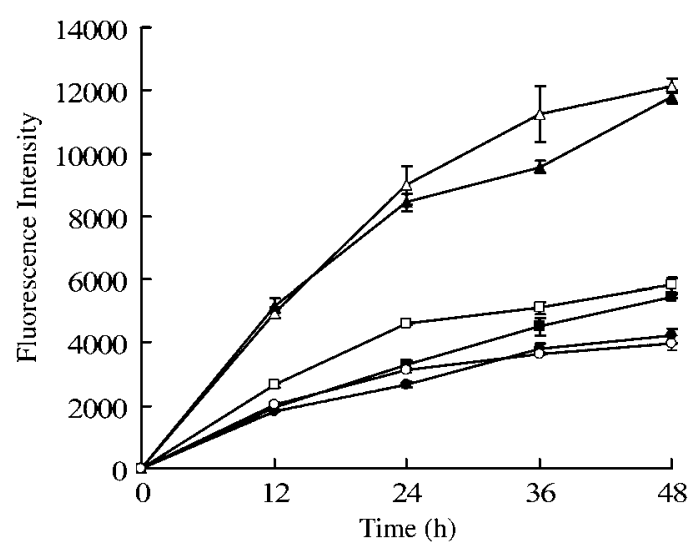

Fig. 2. Changes of the levels of ROS in different strains of C. albicans with time. ROS level was measured using DCFH-DA. Filled circles, RM1000; open squares, JH1U; filled squares, JH1; open triangle, JH2U; filled triangle, JH2; open circles, JHEXP. Each datum point represents means $\pm \mathrm{SD}$ of three independent samples.

indicator of mitochondrial function, of ipf7817 mutant strains. With the deletion of CaIPF7817 in $\mathrm{JH} 2, \Delta \psi_{\mathrm{m}}$ was increased significantly. In the heterozygote $\mathrm{JH} 1$, slight 
increase was observed for $\Delta \psi_{\mathrm{m}}$. No significant difference was found between JHEXP and RM1000 (Fig. 3).

\section{Up-regualtion of some redox-related genes in ipf7817 mutants}

Since the deletion of CaIPF7817 resulted in the dramatic increase of intracellular ROS, we then investigated the expression of some important redox-related genes, including GLR1 (glutathione reductase gene), SOD2 (manganese-superoxide dismutase gene) and TRRI (thioredoxin reductase gene), through real-time RT-PCR analysis. In the JH2, null mutant of ipf7817 GLR1 was found to be up-regulated about 7-fold. SOD2 and TRR1 were also up-regulated in JH2 (Fig. 4).

\section{Increased [GSH]/[GSSG] ratio in ipf7817 mutant}

Given the deletion of CaIPF7817 resulted in the up-regulation of $G L R 1$, we measured the cellular [GSH] $] /[\mathrm{GSSG}$ ] ratio. In ipf7817 null mutant JH2, GSH level was increased by $c a$. $46 \%$ and GSSG level decreased by $c a$. $25 \%$, compared with that of the parental strain. The $[\mathrm{GSH}] /[\mathrm{GSSG}]$

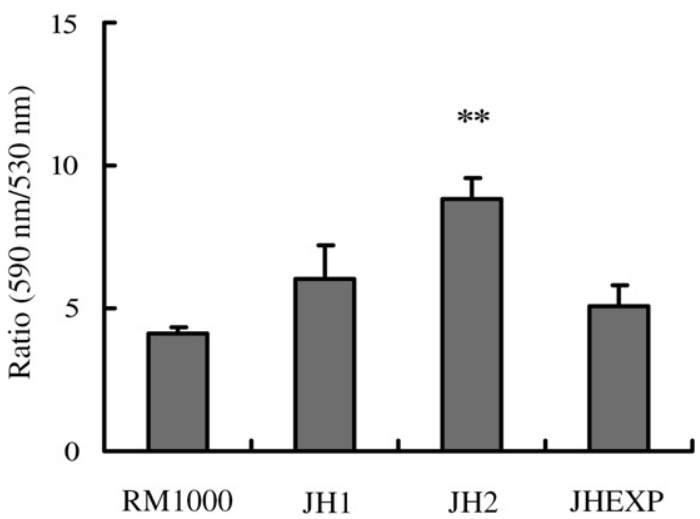

Fig. 3. The mitochondrial membrane potential in different strains of C. albicans. Results are shown as means $\pm \mathrm{SD}$ of three independent experiments. ** indicates $P<0.01$ compared with RM1000

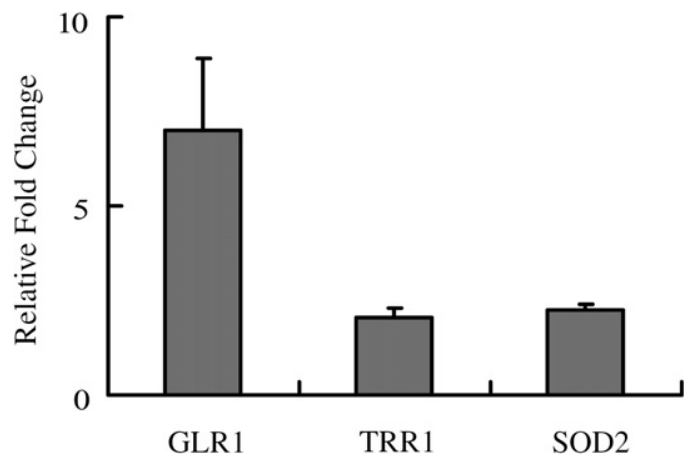

Fig. 4. Changes of expression of some redox-related genes in ipf7817 null mutant determined by real-time RT-PCR. Gene expression is indicated as the fold increase relative to that of the RM1000 strain. Data are shown as means $\pm \mathrm{SD}$ of three independent experiments.
Table 2

Comparison of $[\mathrm{GSH}] /[\mathrm{GSSG}]$ ratio between the mutant and wild-type C. albicans cells

\begin{tabular}{llll}
\hline & GSH $(\mu \mathrm{M})$ & GSSG $(\mu \mathrm{M})$ & GSH/GSSG \\
\hline RM1000 & $17.98 \pm 1.25$ & $1.42 \pm 0.03$ & $12.69 \pm 1.12$ \\
JH1 & $14.41 \pm 2.74$ & $0.83 \pm 0.05$ & $17.53 \pm 4.45$ \\
JH2 & $26.29 \pm 0.40^{\mathrm{a}}$ & $1.07 \pm 0.08$ & $24.51 \pm 2.21^{\mathrm{a}}$ \\
JHEXP & $17.01 \pm 1.35$ & $1.27 \pm 0.13$ & $13.57 \pm 2.50$ \\
\hline
\end{tabular}

${ }^{\text {a }} P<0.05$ compared with RM1000.

ratio in $\mathrm{JH} 2$ increased by approximately 2-fold compared with that of the parental strain (Table 2).

\section{Discussion}

In this work, we interrupted both copies of CaIPF7817 in $C$. albicans and studied the role of CaIPF7817 in cellular redox regulation. Intracellular ROS level, mitochondrial membrane potential, expressions of some important redox-related genes and GSH/GSSG ratio were measured to reveal changes in cellular redox condition after $\mathrm{CaI}$ PF7817 deletion. Without CaIPF7817, the intracellular ROS level was increased; mitochondrial membrane potential was elevated; the expressions of some important redoxrelated genes were up-regulated; and the GSH/GSSG ratio was raised. These changes indicated that CaIPF7817 played roles in the regulation of redox homeostasis of C. albicans.

Mitochondrial respiratory chain is the main source of intracellular ROS and $\Delta \psi_{\mathrm{m}}$ is an important parameter on the redox status of mitochondria. Sophisticated regulation mechanisms exist between intracellular ROS and $\Delta \psi_{\mathrm{m}}[23]$. Because levels of intracellular ROS were affected by $\mathrm{CaI}$ $P F 7817$, we also investigated the effects of CaIPF7817 on $\Delta \psi_{\mathrm{m}}$. The results indicated that $\Delta \psi_{\mathrm{m}}$ was increased significantly with the deletion of CaIPF7817.

The deletion of CaIPF7817 led to a significant increase of intracellular ROS level, but the growth of the null mutant was not affected, indicating that the $\Delta i p f 7817$ cells reached a new intracellular redox balance. We postulated that some reductive molecules and/or reductases might be up-regulated in $\Delta i p f 7817$ cells to counter the increased intracellular ROS. Superoxide dismutases (SODs), which catalyze the direct removal of ROS, play a critical rule in the first line of defense of antioxidants [24,25]. The glutathione and thioredoxin systems are also very important in the redox homeostasis [26,27], in which glutathione reductase $(G L R l)$ and thioredoxin reductase $(T R R l)$ are the key enzymes [28]. Real-time RT-PCR showed that all these three genes, SOD2, GLR1, TRR1, were significantly upregulated in ipf7817 null mutant $\mathrm{JH} 2$. The up-regulation of these genes is agreement with our prediction that antioxidation system should be activated in $\Delta i p f 7817$ cells to balance the increased intracellular levels of ROS. Moreover, the increased GSH/GSSG ratio also supported this prediction. Therefore the increase of ROS production and activa- 
tion of the antioxidation system led to a new intracellular redox balance in $\Delta i p f 7817$ cells.

CaIPF7817 is highly up-regulated during oxidative stress $[11,12]$, implying that it has a role in oxidative stress tolerance. We therefore, examined the sensitivity of the ipf7817 null mutant to $\mathrm{H}_{2} \mathrm{O}_{2}$. Contrary to our expectation, no difference was found between the growth of the ipf 7817 null mutant and that of the parental strain in the presence of $\mathrm{H}_{2} \mathrm{O}_{2}$ (data not shown). It was reported that, $\mathrm{CaI}$ PF7817 was not uniquely up-regulated upon extracellular oxidative damage $[11,12]$. Our $\mathrm{H}_{2} \mathrm{O}_{2}$ sensitivity experiment showed that CaIPF7817 was not essential for the tolerance of extracellular oxidants, suggesting that CaIPF7817 was mainly involved in the regulation of intracellular redox homeostasis in C. albicans, especially during the normal aerobic metabolism of $C$. albicans.

\section{Acknowledgments}

This work was funded by a grant from the National Natural Science Foundation of China (30500628) and the National 973 Program of China (2005CB523105). We thank J. Pla for kindly providing us the C. albicans strain RM1000 and W.A. Fonzi for plasmid p5921.

\section{References}

[1] M.D. Richardson, Changing patterns and trends in systemic fungal infections, J. Antimicrob. Chemother. 56 (Suppl. 1) (2005) i5-i11.

[2] G. Garber, An overview of fungal infections, Drugs 61 (Suppl. 1) (2001) 1-12.

[3] N. Ivanovska, M. Hristova, S. Philipov, Immunosuppression and recovery of drug-impaired host resistance against Candida albicans infection by oxoglaucine, Pharmacol. Res. 41 (2000) 101-107.

[4] A. Ikner, K. Shiozaki, Yeast signaling pathways in the oxidative stress response, Mutat. Res. 569 (2005) 13-27.

[5] I.W. Dawes, Yeast stress responses, in: J.R. Dickinson, M. Schweizer (Eds.), The Metabolism and Molecular Physiology of Saccharomyces cerevisiae, second ed., CRC Press, Boca Raton, FL, 2004, pp. 376-438.

[6] D.J. Jamieson, Oxidative stress responses of the yeast Saccharomyces cerevisiae, Yeast 14 (1998) 1511-1527.

[7] R. Ivarsson, R. Quintens, S. Dejonghe, K. Tsukamoto, P. In 't Veld, E. Renstrom, F.C. Schuit, Redox control of exocytosis: regulatory role of NADPH, thioredoxin, and glutaredoxin, Diabetes 54 (2005) 2132-2142.

[8] A.J. Phillips, I. Sudbery, M. Ramsdale, Apoptosis induced by environmental stresses and amphotericin B in Candida albicans, Proc. Natl. Acad. Sci. USA 100 (2003) 14327-14332.

[9] G.L. Wheeler, C.M. Grant, Regulation of redox homeostasis in the yeast Saccharomyces cerevisiae, Physiol. Plant 120 (2004) 12-20.

[10] W. Dröge, Free radicals in the physiological control of cell function, Physiol. Rev. 82 (2002) 47-95.

[11] Y. Wang, Y.Y. Cao, X.M. Jia, Y.B. Cao, P.H. Gao, X.P. Fu, K. Ying, W.S. Chen, Y.Y. Jiang, Caplp is involved in multiple pathways of oxidative stress response in Candida albicans, Free Radic. Bio. Med. 40 (2006) 1201-1209.

[12] H. Kusch, S. Engelmann, D. Albrecht, J. Morschhauser, M. Hecker, Proteomic analysis of the oxidative stress response in Candida albicans, Proteomics 7 (2007) 686-697.

[13] Z. Xu, Y.B. Cao, J.D. Zhang, Y.Y. Cao, P.H. Gao, D.J. Wang, X.P. Fu, K. Ying, W.S. Chen, Y.Y. Jiang, cDNA array analysis of the differential expression change in virulence-related genes during the development of resistance in Candida albicans, Acta Biochim. Biophys. Sin. 37 (2005) 463-472.

[14] W.A. Fonzi, M.Y. Irwin, Isogenic strain construction and gene mapping in Candida albicans, Genetics 134 (1993) 717-728.

[15] T. Umeyama, Y. Nagai, M. Niimi, Y. Uehara, Construction of FLAG tagging vectors for Candida albicans, Yeast 19 (2002) 611-618.

[16] D. Sanglard, F. Ischer, M. Monod, J. Bille, Susceptibilities of Candida albicans multidrug transporter mutants to various antifungal agents and other metabolic inhibitors, Antimicrob. Agents Chemother. 40 (1996) 2300-2305.

[17] J.D. Boeke, F. LaCroute, G.R. Fink, A positive selection for mutants lacking orotidine- $5^{\prime}$-phosphate decarboxylase activity in yeast: 5fluoro-orotic acid resistance, Mol. Gen. Genet. 197 (1984) 345-346.

[18] D. Kobayashi, K. Kondo, N. Uehara, S. Otokozawa, N. Tsuji, A. Yagihashi, N. Watanabe, Endogenous reactive oxygen species is an important mediator of miconazole antifungal effect, Antimicrob. Agents Chemother. 46 (2002) 3113-3117.

[19] A. Hassouna, M. Loubani, B.M. Matata, A. Fowler, N.B. Standen, M. Galinanes, Mitochondrial dysfunction as the cause of the failure to precondition the diabetic human myocardium, Cardiovasc. Res. 69 (2006) 450-458.

[20] C. Vandeputte, I. Guizon, I. Genestie-Denis, B. Vannier, G. Lorenzon, A microtiter plate assay for total glutathione and glutathione disulfide contents in cultured/isolated cells: performance study of a new miniaturized protocol, Cell Biol. Toxicol. 10 (1994) 415-421.

[21] A. Martirosyan, S. Leonard, X. Shi, B. Griffith, P. Gannett, J. Strobl, Actions of a histone deacetylase inhibitor, NSC3852, link reactive oxygen species to cell differentiation and apoptosis in MCF-7 human mammary tumor cells, J. Pharmacol. Exp. Ther. 317 (2006) 546-552.

[22] L. Simon, G. Szilagyi, Z. Bori, G. Telek, K. Magyar, Z. Nagy, Low dose (-)deprenyl is cytoprotective: it maintains mitochondrial membrane potential and eliminates oxygen radicals, Life Sci. 78 (2005) 225-231.

[23] D.G. Nicholls, S.L. Budd, Mitochondria and neuronal survival, Physiol. Rev. 80 (2000) 315-357.

[24] E. Luk, M. Yang, L.T. Jensen, Y. Bourbonnais, V.C. Culotta, Manganese activation of superoxide dismutase 2 in the mitochondria of Saccharomyces cerevisiae, J. Biol. Chem. 280 (2005) 22715-22720.

[25] D.D. Tyler, Polarographic assay and intracellular distribution of superoxide dismutase in rat liver, Biochem. J. 147 (1975) 493-504

[26] O. Carmel-Hare, G. Storz, Roles of the glutathione-and thioredoxindependent reduction systems in the Escherichia coli and Saccharomyces cerevisiae responses to oxidative stress, Annu. Rev. Microbiol. 54 (2000) 439-461.

[27] C.M. Grant, Role of the glutathione/glutaredoxin and thioredoxin systems in yeast growth and response to stress conditions, Mol. Microbiol. 39 (2001) 533-541.

[28] E.W. Trotter, C.M. Grant, Non-reciprocal regulation of the redox status of the glutathione-glutaredoxin and thioredoxin systems, EMBO Rep. 4 (2003) 184-188. 